01

\title{
Исследование кинетических параметров схемы лазерной фотоионизации лютеция
}

\author{
(ㄱ А.Б. Дьячков, А.А. Горкунов, А.В. Лабозин, С.М. Миронов, В.Я. Панченко, В.А. Фирсов, Г.О. Цветков \\ Национальный исследовательский центр „Курчатовский институт“, \\ 123182 Москва, Россия \\ e-mail: Tsvetkov_GO@nrcki.ru
}

Поступила в редакцию 26.09.2019 г.

В окончательной редакции 26.09.2019 г.

Принята к публикации 29.11.2019 г.

Разработка лазерного фотоионизационного метода получения радионуклида ${ }^{177} \mathrm{Lu}$ для применения в медицине требует знания интенсивностей светового насыщения по каждой ступени схемы фотоионизации лютеция $\left(5 d 6 s^{2}{ }^{2} D_{3 / 2}-5 d 6 s 6 p^{4} F_{5 / 2}^{\circ}-5 d 6 s 7 s^{4} D_{3 / 2}-\left(53375 \mathrm{~cm}^{-1}\right)_{1 / 2}^{\circ}\right)$ с учетом используемых компонент сверхтонкой структуры переходов. В работе экспериментально определены эффективные сечения возбуждения различных сверхтонких компонент переходов для изотопов ${ }^{175} \mathrm{Lu},{ }^{176} \mathrm{Lu},{ }^{177} \mathrm{Lu}$ и ${ }^{177 m} \mathrm{Lu}$ излучением импульсных перестраиваемых по длине волны лазеров на красителях, накачиваемых лазерами на парах меди.

Ключевые слова: лазерная фотоионизация, сверхтонкая структура, ${ }^{177} \mathrm{Lu}$, лазерное разделение изотопов.

DOI: 10.21883/OS.2020.03.49055.277-19

\section{Введение}

В настоящее время считается, что радиоизотоп ${ }^{177} \mathrm{Lu}$ имеет все шансы стать наиболее применяемым в медицине к 2020 г. благодаря удачному сочетанию невысокой энергии бета-излучения, периода полураспада 6.64 суток и спектра сопутствующего гамма-излучения. Получение

${ }^{177} \mathrm{Lu}$ основано на нейтронном облучении предварительно обогащенных изотопов ${ }^{176} \mathrm{Lu}$ или ${ }^{176} \mathrm{Yb}$. В первом случае образуется значительное количество долгоживущего изомера ${ }^{177 m} \mathrm{Lu}$, который является источником радиоактивных отходов. Недостатком второго метода является низкое сечение поглощения нейтронов. Использование лазерного фотоионизационного метода предоставляет возможность модифицировать изотопный состав природного лютеция, облученного в реакторе, осуществляя очистку от изомера при выделении ${ }^{177} \mathrm{Lu} \mathrm{и/или} \mathrm{выде-}$ ление изомера ${ }^{177 m} \mathrm{Lu}$ для дальнейшего использования в независимом генераторе ${ }^{177} \mathrm{Lu}$ [1]. Указанные возможности непосредственно вытекают из свойств трехступенчатой схемы фотоионизации лютеция: $5 d 6 s^{2}{ }^{2} D_{3 / 2}-$ $-5 d 6 s 6 p^{4} F_{5 / 2}^{\circ}-5 d 6 s 7 s^{4} D_{3 / 2}-\left(53375 \mathrm{~cm}^{-1}\right)_{1 / 2}^{\circ}$, спектроскопия которой для различных изотопов детально исследована в работах [2,3]. Особенностью схемы является то, что все уровни имеют сверхтонкую структуру (СТC), что приводит к появлению большого числа (44) возможных каналов фотоионизации конкретного изотопа лютеция, отличающихся не только длинами волн, но и вероятностями переходов. При этом каналы различных изотопов могут накладываться друг на друга, что приводит к тому, что селективность фотоионизации по различным каналам сильно отличается [4]. При осуществлении селективной фотоионизации важен не только выбор канала, но и правильный выбор интенсивностей лазеров всех трех ступеней с тем, чтобы добиться максимальной селективности и эффективности фотоионизации.

Настоящая работа посвящена исследованию процессов насыщения переходов трехступенчатой схемы фотоионизации с целью определения эффективных сечений возбуждения различных компонент СТС для изотопов ${ }^{175} \mathrm{Lu},{ }^{176} \mathrm{Lu},{ }^{177} \mathrm{Lu}$ и ${ }^{177 m} \mathrm{Lu}$.

Сечение перехода между сверхтонкой компонентой $F$ уровня 1 и компонентой $F$ уровня 2 можно записать как

$$
\sigma_{F \dot{F}}=C(F, J, \grave{F}, \grave{J}, I) \tilde{\sigma}_{1 / 2},
$$

где $\tilde{\sigma}_{1 / 2}-$ полное сечение перехода 1-2, $C(F, J, \grave{F}, \grave{J}, I)$ - коэффициент, зависящий от угловых моментов, участвующих в переходе: $F, J, I-$ полный угловой момент атома, электронный момент атома и спин ядра соответственно. Формулы для сечений с угловыми коэффициентами в явном виде приведены в работе [5] (см. приложение).

\section{Экспериментальная установка}

Исследования проводились на установке, предназначенной для экспериментов по лазерной фотоионизационной спектроскопии в узких коллимированных атомных пучках с возможностью определения изотопного состава фотоионов. Установка состоит из вакуумной камеры с системой откачки, испарителем и квадрупольным масс-спектрометром МС-7302. Атомный пучок, формируемый испарителем, попадает в источник ионов масс-спектрометра. Для резонансного возбуждения и 
ионизации атомов используется излучение трех импульсных одномодовых лазеров на красителях (ЛК) со спектральной шириной линии генерации $100-150 \mathrm{MHz}$ (FWHM), накачиваемых лазерами на парах меди. Лазерный луч пересекает атомный пучок непосредственно в ионизационной камере источника ионов. Направления атомного, лазерного пучков и ионно-оптической оси масс-спектрометра взаимно ортогональны. Регистрация ионов в масс-спектрометре производится вторичноэлектронным умножителем (ВЭУ). Для контроля длины волны генерации ЛК используются прецизионные измерители длины волны.

Управление установкой и регистрация данных осуществляется в режиме on-line. Программное обеспечение, реализованное в среде LabView (National Instruments), выполняет необходимые действия: принимает данные от измерителей длин волн, сигналы с ВЭУ и с помощью блока сопряжения изменяет управляющее напряжение, перестраивая либо стабилизируя каждый ЛК по длине волны генерации. Технические параметры и особенности экспериментальной установки детально изложены в работе [6].

ЛК первой и второй ступеней схемы фотоионизации состояли только из задающих генераторов ЛК с выходной средней мощностью $\sim 300 \mathrm{~mW}$. В лазере третьей ступени был использован один усилитель ЛК с подъемом выходной средней мощности до 4-5 W.

Лучи первых двух ступеней телескопировались до поперечного размера $\varnothing=10 \mathrm{~mm}$ и коллимировались. Из-за близости длин волн (540 и $535 \mathrm{~nm})$ данные лучи пространственно сводились на полупрозрачном зеркале. Сведение с лучом третьей ступени $(618 \mathrm{~nm})$ производилось на зеркале с дихроичным диэлектрическим покрытием. Система поворотных зеркал направляла единый трехцветный луч в масс-спектрометрическую камеру. Для уверенного насыщения линий ступени фотоионизации луч третьей ступени фокусировался в зону испарения с расстояния $20 \mathrm{~m}$ в поперечный размер $\varnothing \sim 5 \mathrm{~mm}$. Для временной синхронизации импульсов ЛК (задержка импульсов друг относительно друга) в трактах лучей использовались соответствующие пространственные линии задержки.

В масс-спектрометрической камере луч проходил через диафрагму с диаметром отверстия $\varnothing=2 \mathrm{~mm}$, которая была установлена непосредственно перед зоной испарения. Диафрагма вырезала наиболее однородную центральную часть лазерного луча. Плотность средней мощности ЛК в зоне взаимодействия света и пара рассчитывалась по средней мощности прошедшего диафрагму излучения на выходе из камеры.

Методика экспериментов по определению сечений переходов состояла в том, что длины волн всех трех ступеней настраивались на определенные компоненты и стабилизировались. Масс-спектрометр настраивался на регистрацию фотоионов выделенного изотопа, а сигнал с ВЭУ оцифровывался и записывался в файл. В ходе

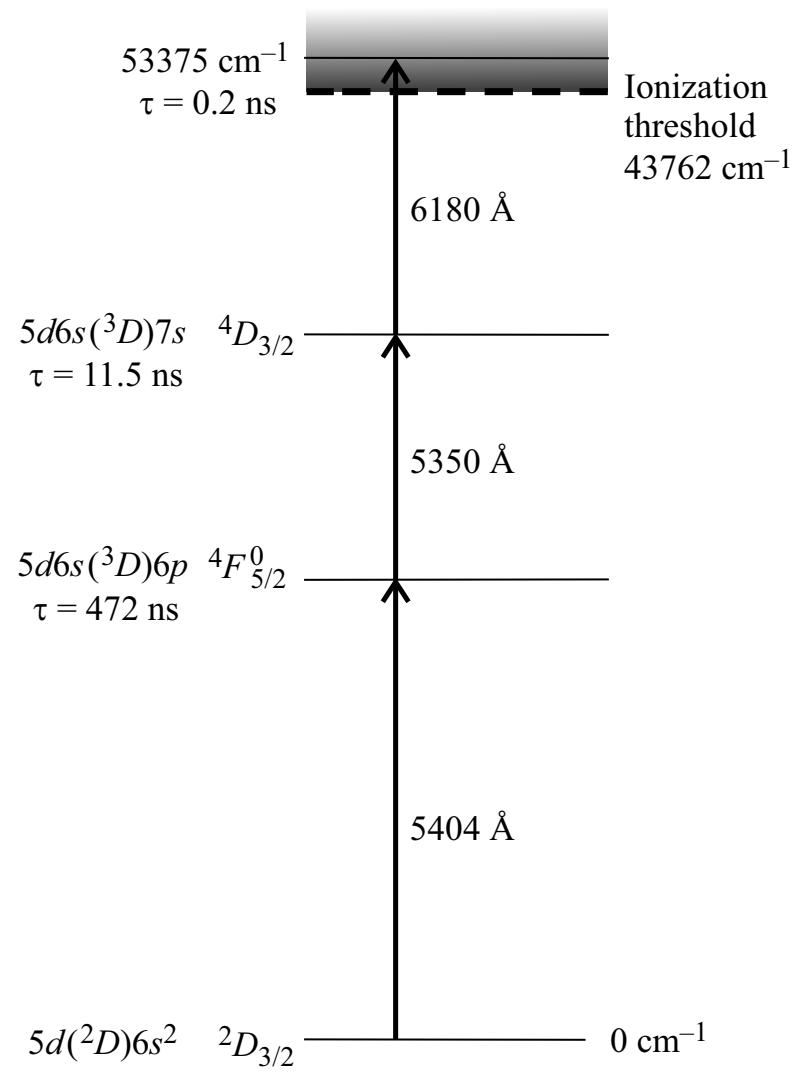

Pис. 1. Схема трехступенчатой лазерной фотоионизации LuI.

эксперимента интенсивность лазерного излучения выделенного перехода изменялась ступенчато по заранее определенному графику.

Для изменения лазерной мощности использовался набор ослабителей (плоскопараллельных кварцевых окон, $\varnothing=50 \mathrm{~mm}$, с широкополосным диэлектрическим покрытием и коэффициентами пропускания $\sim 0.1,0.2, \ldots 1)$. Окна (10 шт) устанавливались на вращающемся диске. На пути одного из лучей ЛК устанавливалось два диска-ослабителя, что позволяло оперативно регулировать уровень средней мощности ЛК в диапазоне 0.01-1W. Высокая параллельность входных и выходных поверхностей окон исключала смещение прошедшего луча. Качественное просветляющее диэлектрическое покрытие на выходной поверхности окон предельно снижало модуляцию профиля интенсивности луча за счет интерференции (в особенности для окон с низким пропусканием) и соответственно исключало ошибку при расчете плотности мощности. Каждый ослабитель был предварительно откалиброван по пропусканию на длинах волн каждой ступени.

В экспериментах исследовалась зависимость фотоионного тока от интенсивности ЛК, настроенного на СТС-линию одного из переходов схемы фотоионизации лютеция $5 d 6 s^{22} D_{3 / 2}-5 d 6 s 6 p^{4} F_{5 / 2}^{\circ}-5 d 6 s 7 s^{4} D_{3 / 2}-$ $-\left(53375 \mathrm{~cm}^{-1}\right)_{1 / 2}^{\circ}$ (рис. 1$)$. 


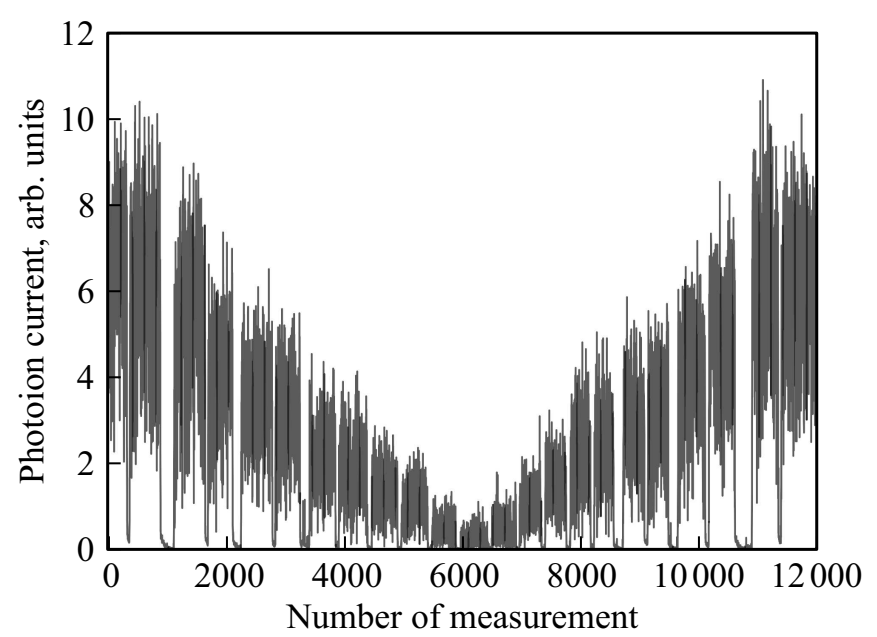

Рис. 2. Запись фотоионного сигнала в канале ионизации ${ }^{176} \mathrm{Lu}$ $F: 8.5 \rightarrow 7.5 \rightarrow 8.5 \rightarrow 7.5$ при последовательном внесении в луч лазера 1 ступени калиброванных ослабителей. Провалы до нуля соответствуют перекрытию луча в момент смены окон ослабителя.

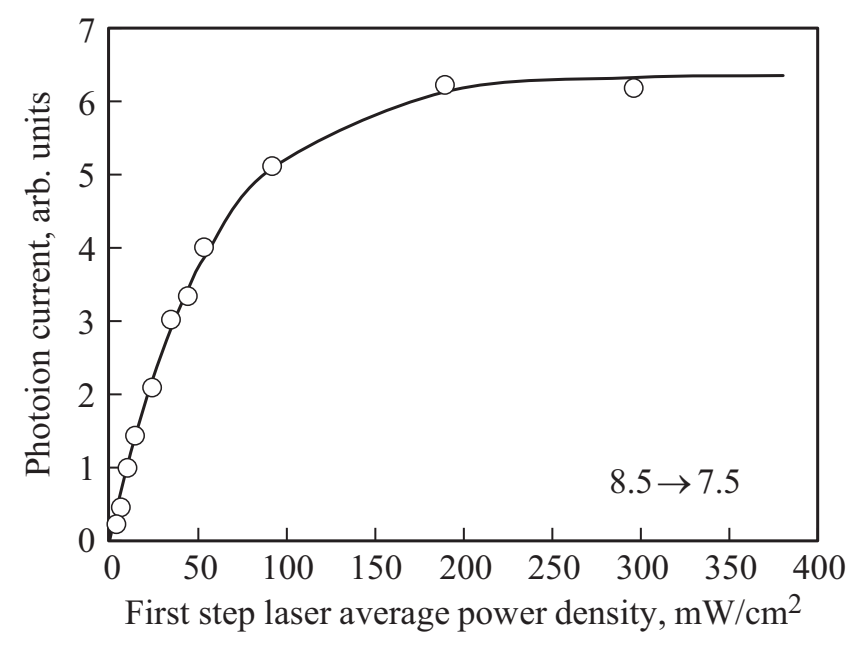

Рис. 3. Зависимость фотоионного сигнала от средней плотности мощности лазерного излучения первого перехода ${ }^{176} \mathrm{Lu}$ $F=8.5 \rightarrow F=7.5$ : экспериментальные точки и аппроксимирующая кривая с эффективным сечением первого перехода $\sigma_{8.5-7.5} \approx 3.2 \cdot 10^{-14} \mathrm{~cm}^{2}$.

Измерения мощности в луче ЛК проводилось off-line в положении максимального пропускания ослабителей. В ходе записи сигнала фотоионизации производилось пошаговое снижение мощности ЛК сменой ослабителей в заранее определенном порядке (вращением дисков). Длительность выдержки постоянного значения интенсивности составляла приблизительно $2 \mathrm{~s}$. В среднем в экспериментах использовалось от 11 до 13 комбинаций пропускания. После достижения минимального пропускания процедура повторялась в обратном порядке (рис. 2), и производилось повторное измерение средней мощности ЛК.

\section{Обработка экспериментальных результатов}

Эффективное сечение возбуждения переходов определялось методом аппроксимации экспериментальной зависимости теоретической кривой.

Исследование переходов первой и второй ступеней осуществлялось последовательными лазерными импульсами, не пересекавшимися во времени, с тем чтобы избежать влияния многофотонных процессов. В этих условиях внутри каждого импульса атомная система может рассматриваться как двухуровневая, и теоретическая зависимость может быть получена из решения системы двух кинетических уравнений

$$
\begin{gathered}
\frac{d N_{i}}{d t}=-N_{i} w_{i k}+N_{k} w_{k i}, \\
\frac{d N_{k}}{d t}=N_{i} w_{i k}-N_{k}\left(w_{k i}+a\right),
\end{gathered}
$$

где $N_{i}$ и $N_{k}$ - число частиц в нижнем и верхнем состояниях соответственно, $w_{i k}$ - частота лазерноиндуцированных переходов из состояния $i$ в состояние $k\left(w_{i k}=\frac{I_{i k}}{h v_{i k}}\right)$, где $I-$ интенсивность лазерного излучения $\left(\mathrm{W} / \mathrm{cm}^{2}\right), \sigma_{i k}-$ сечение перехода $\left(\mathrm{cm}^{2}\right)$, $v$ - частота перехода $i k) ; a$ - частота распадов из состояния $k$ в метастабильные состояния (при $k=2$ частота $a=1 / 472 \mathrm{~ns}=2.1$ МГц, при $k=3$ частота $a=1 / 11.5 \mathrm{Hc}=87 \mathrm{MHz})$. В данной записи мы пренебрегли спонтанными распадами, обратными вынужденным лазерно-индуцированным переходам, а также распадом нижнего уровня $i$, поскольку при $i=1$ распад основного состояния отсутствует, а при $i=2$ время жизни первого возбужденного состояния (472 ns) существенно превышает длительность лазерного импульса $20 \mathrm{~ns}$. Решение этой системы для $N_{k}$ в условиях прямоугольного лазерного импульса длительностью $\tau$ ( $I=$ const при $0<t<\tau$ ) и начальных условиях (при $t=0) N_{k}=0, d N_{k} / d t=N_{0} w_{i k}$ имеет вид

$$
\begin{gathered}
N_{k}(\tau)=\frac{N_{0} w_{i k}}{A}\left[\exp \left(\frac{-B+A}{2} \tau\right)-\exp \left(\frac{-B-A}{2} \tau\right)\right], \\
A=\left(\frac{g_{i}+g_{k}}{g_{k}} w_{i k}+a\right), \\
B=\sqrt{\left(\frac{g_{i}+g_{k}}{g_{k}} w_{i k}+a\right)^{2}-4 a w_{i k},}
\end{gathered}
$$

где $g_{i}$ и $g_{k}$ - статистические веса состояний $i$ и $k$ соответственно.

Интенсивность $I\left(\mathrm{~W} / \mathrm{cm}^{2}\right)$ лазерного излучения определялась по формуле

$$
I=\frac{P}{f \tau}
$$

где $P\left(\mathrm{~W} / \mathrm{cm}^{2}\right)$ - средняя плотность мощности лазерного излучения, измеряемая в эксперименте, $f-$ 

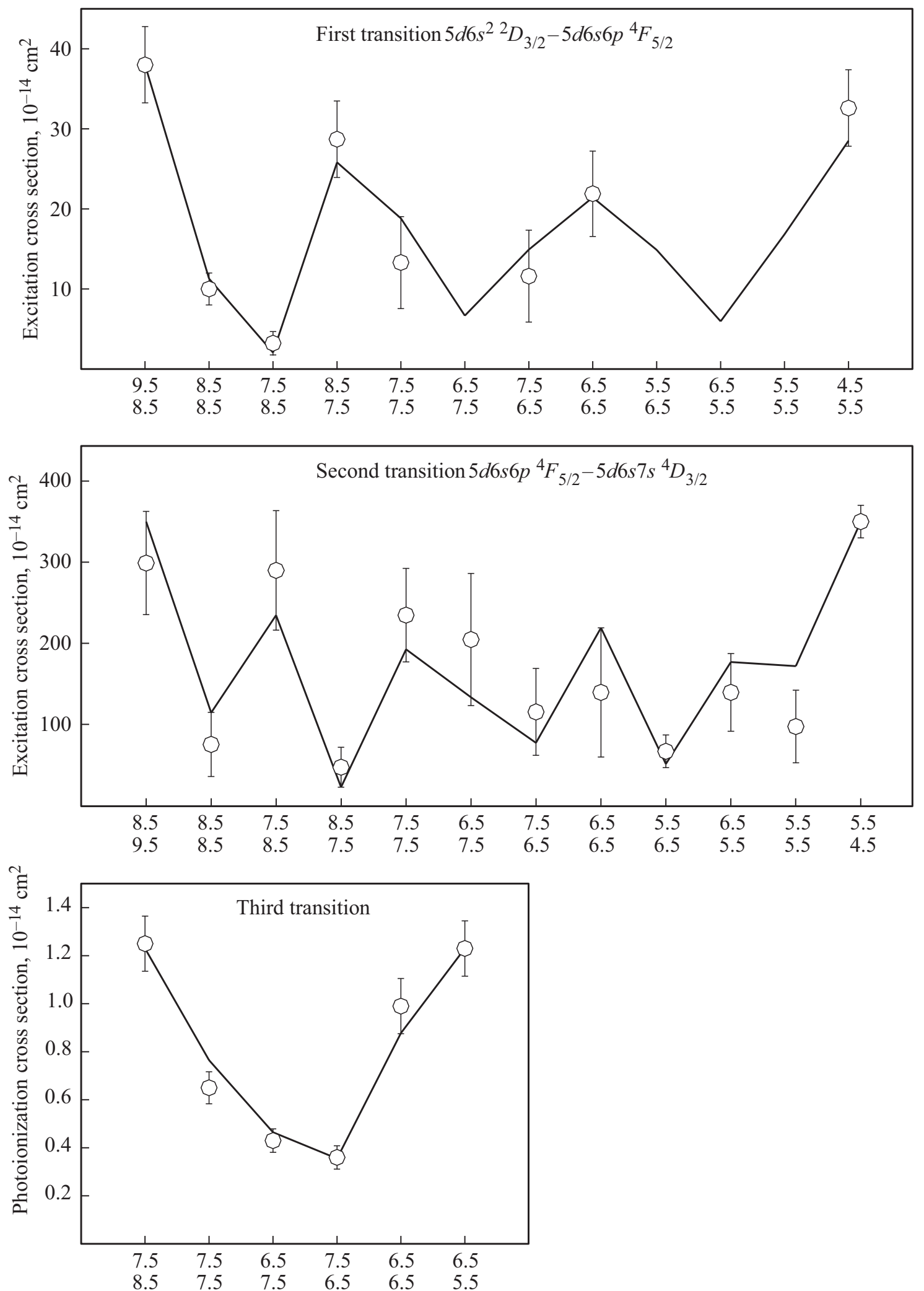

Рис. 4. Эффективные сечения компонент переходов трехступенчатой схемы $5 d 6 s^{22} D_{3 / 2}-5 d 6 s 6 p^{4} F_{5 / 2}^{\circ}-5 d 6 s 7 s^{4} D_{3 / 2}-$ $-\left(53375 \mathrm{~cm}^{-1}\right)_{1 / 2}^{\circ}$ изотопа ${ }^{176} \mathrm{Lu}$. Сплошная ломаная линия соединяет расчетные значения с полным сечением $\tilde{\sigma}_{I 176}=5.1 \cdot 10^{-13} \mathrm{~cm}^{2}, \tilde{\sigma}_{I I 176}=3.5 \cdot 10^{-12} \mathrm{~cm}^{2}, \tilde{\sigma}_{I I I 176}=1.2 \cdot 10^{-14} \mathrm{~cm}^{2}$ первого, второго и третьего переходов соответственно. 


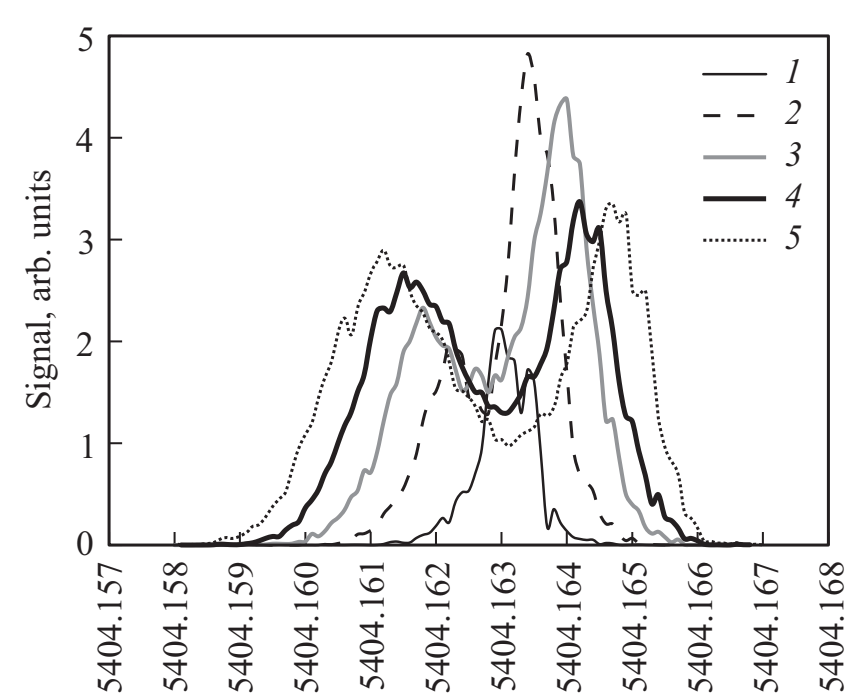

First step laser wavelength, $\AA$

Рис. 5. Фотоионный ток ${ }^{175} \mathrm{Lu}$ в канале $2-1-2-3$ при разных интенсивностях ЛК второй ступени. $1-0.3 \mathrm{мBT} / \mathrm{cm}^{2}, 2-$ $3 \mathrm{MBT} / \mathrm{cm}^{2}, 3-8 \mathrm{MBT} / \mathrm{cm}^{2}, 4-16 \mathrm{MBT} / \mathrm{cm}^{2}, 5-29 \mathrm{MBT} / \mathrm{cm}^{2}$.

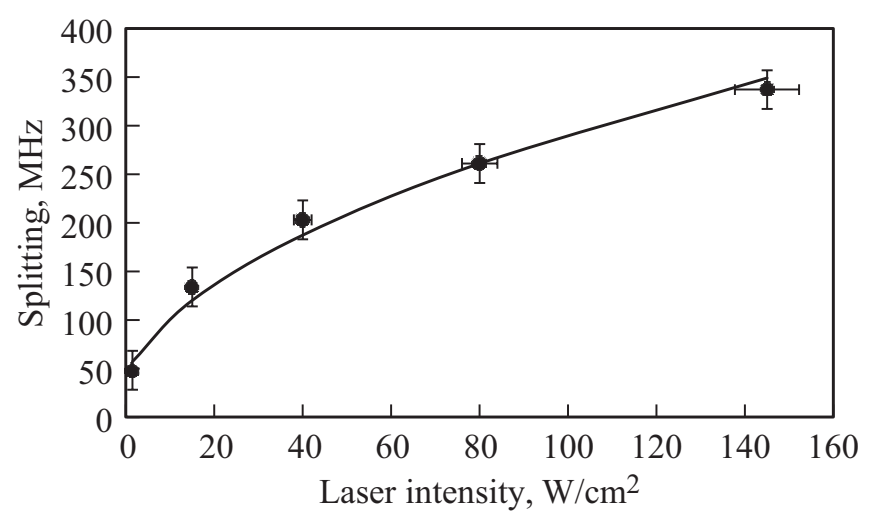

Рис. 6. Зависимость величины расщепления первого перехода ${ }^{175} \mathrm{Lu}$ (компонента 2-1) от интенсивности лазерного излучения второго перехода (компонента 1-2). Сплошная линия расчет при формуле (10) с коэффициентом Эйнштейна второго перехода $A_{2-1}=8(1) \cdot 10^{5} 1 / \mathrm{s}$ и отстройкой частоты второго перехода $\Delta \lambda_{23}=45(5) \mathrm{MHz}$.

частота следования лазерных импульсов $(10 \mathrm{kHz})$ и $\tau-$ длительность импульса (20 ns).

На рис. 3 представлена экспериментальная зависимость для первого перехода ${ }^{176} \mathrm{Lu} F: 8.5 \rightarrow 7.5 \mathrm{c}$ аппроксимированной зависимостью по формуле (4) с эффективным сечением первого перехода $\sigma_{8.5-7.5} \approx$ $\approx 3.2 \cdot 10^{-14} \mathrm{~cm}^{2}$.

Исследование третьего перехода проводилось в совмещенных во времени импульсах, поскольку многофотонные процессы не оказывают влияние на точность измерений сечения фотоионизации вследствие его малости по сравнению с сечениями первого и второго переходов. Экспериментальная зависимость аппроксими-
Значения полных сечений переходов

\begin{tabular}{c|c|c|c}
\hline Изотоп & $\tilde{\sigma}_{I}, \mathrm{~cm}^{2}$ & $\tilde{\sigma}_{I I}, \mathrm{~cm}^{2}$ & $\tilde{\sigma}_{I I I}, \mathrm{~cm}^{2}$ \\
\hline${ }^{175} \mathrm{Lu},{ }^{177} \mathrm{Lu}$ & $4.5(1) \cdot 10^{-13}$ & $1.6(3) \cdot 10^{-12}$ & $1.2(2) \cdot 10^{-14}$ \\
\hline${ }^{176} \mathrm{Lu}$ & $5(1) \cdot 10^{-13}$ & $3.5(5) \cdot 10^{-12}$ & $1.5(3) \cdot 10^{-14}$ \\
\hline${ }^{177 m} \mathrm{Lu}$ & $5(1) \cdot 10^{-13}$ & $2.3(3) \cdot 10^{-12}$ & $2.1(3) \cdot 10^{-14}$
\end{tabular}

ровалась функцией [7]

$$
\frac{N_{i}}{N_{0}}=\frac{w_{34}}{\left(w_{34}+a_{3 M}\right)}\left[1-\exp \left(-\frac{g_{3}\left(w_{34}+a_{3 M}\right)}{\left(g_{1}+g_{2}+g_{3}\right)} \tau\right)\right] .
$$

Здесь $g_{1}, g_{2}$ и $g_{3}-$ статистические веса начального, первого и второго возбужденных состояний соответственно, $w_{34}$ - частота лазерно-индуцированных переходов из второго возбужденного состояния в автоионизационное состояние $\left(w_{34}=\frac{I \sigma_{34}}{h v_{34}}\right.$, где $I-$ интенсивность лазерного излучения третьей ступени $\left(\mathrm{W} / \mathrm{cm}^{2}\right), \sigma_{34}-$ сечение третьего перехода $\left(\mathrm{cm}^{2}\right), v_{34}$ - частота третьего перехода); $a_{3 м}$ - частота распадов из второго возбужденного состояния в метастабильные состояния $(87 \mathrm{MHz})$.

\section{Результаты и обсуждение}

Следует отметить, что сечения не всех линий СТС первого перехода могли быть измерены по описанной выше методике. Для некоторых линий на расстоянии $150-400 \mathrm{MHz}$ находятся линии возбуждения с других нижних подуровней [3]. Возбуждение и ионизация с данных подуровней при увеличении интенсивности оказывали заметное влияние на ход зависимости фотоионного тока и делали невозможным определение индивидуальных сечений. К таким линиям относятся пары 2-3, 4-3 и $3-4,4-4$ для изотопов ${ }^{175} \mathrm{Lu}$ и ${ }^{177} \mathrm{Lu}, 5.5-6.5,7.5-6.5$ и $5.5-5.5,6.5-5.5$ для ${ }^{176} \mathrm{Lu}, 10-11,12-11$ и $11-12$, $12-12$ для ${ }^{177 m} \mathrm{Lu}$.

На рис. 4 представлены результаты определения эффективных сечений для компонент переходов изотопа ${ }^{176} \mathrm{Lu}$. Сплошная ломаная линия соединяет значения, рассчитанные по формуле (1) с полными сечениями $\tilde{\sigma}_{I 176}=5.1 \cdot 10^{-13} \mathrm{~cm}^{2}, \tilde{\sigma}_{I I 176}=3.5 \cdot 10^{-12} \mathrm{~cm}^{2}$ и $\tilde{\sigma}_{I I I} 176=1.2 \cdot 10^{-14} \mathrm{~cm}^{2}$ первого, второго и третьего переходов, которые соответствуют минимальной сумме квадратов отклонений от экспериментальных результатов. Из приведенных результатов видно, что экспериментальные соотношения сечений компонент находятся в хорошем согласии с расчетом. Аналогичным образом были найдены эффективные сечения компонент, а затем и полные эффективные сечения для всех ступеней схемы фотоионизации для изотопов ${ }^{175} \mathrm{Lu}$, ${ }^{177} \mathrm{Lu}$ и ${ }^{177 m} \mathrm{Lu}$ (таблица). Значения сечений ${ }^{177} \mathrm{Lu} \mathrm{не}$ отличались от соответствующих значений для ${ }^{175} \mathrm{Lu}$ в пределах экспериментальных погрешностей, что можно 
объяснить подобием СТС-данных изотопов с одинаковыми спинами ядер $(I=7 / 2)$ и близкими (в пределах $3 \%)$ значениями магнитных дипольных моментов $[8,9]$ : $\mu^{177}=2.2384(14) \mu_{N}[8], \mu^{175}=2.2323(11) \mu_{N}[9]$.

Следует отметить, что в результате экспериментов получены эффективные сечения, которые являются характеристиками не только атомной системы, но и той лазерной системы, которая использовалась для возбуждения переходов (спектральная ширина генерации ЛК в отдельном импульсе, временная форма импульса). В связи с этим для того чтобы исключить влияние особенностей лазерной системы и связать эффективные сечения с параметрами атомной системы, были проведены эксперименты по определению частоты Раби [10] осцилляции населенностей уровней схемы фотоионизации, которая связана с коэффициентом Эйнштейна выражением

$$
F_{\text {Rik }}=1.24 \cdot 10^{8} \sqrt{\left.\lambda^{3}[\AA] I_{i k}\left[\mathrm{~W} / \mathrm{cm}^{2}\right] g_{i} A_{i k}[1 / \mathrm{s}]\right)},
$$

где $\lambda$ - длина волны в ангстремах, $I-$ интенсивность лазерного излучения на переходе $i k, g_{i}-$ статистический вес нижнего состояния перехода и $A-$ коэффициент Эйнштейна перехода, на котором измеряется частота Раби. Теоретические исследования показывают, что при одновременном воздействии на трехуровневую атомную систему лазерного излучения с частотами $v_{12}$ и $v_{23}$, соответствующими первому и второму переходу, населенности уровней испытывают колебания с характерными частотами $F_{R 12}$ и $F_{R 23}$. При $F_{R 12} \ll F_{R 23}$ происходит расщепление первого перехода, и при сканировании $v_{12}$ наблюдаются два пика амплитуды заселенности второго возбужденного состояния 3 при

$$
v_{12}=\sqrt{\left(\Delta v_{23}^{2}+4 F_{R 23}^{2}\right)},
$$

где $\Delta v_{23}$ - отстройка лазерного излучения от центра второго перехода $(\mathrm{MHz}), F_{R 23}$ - частота Раби второго перехода (MHz) [11,12].

На рис. 5 представлены результаты сканирования длины волны первого перехода ${ }^{175} \mathrm{Lu}$ на компоненте 2-1 при различной интенсивности лазерного излучения второй ступени на компоненте 1-2. Амплитуда заселенности второго возбужденного состояния определялась по фотоионному току в результате фотоионизации импульсом третьей ступени (компонента 2-3), задержанным относительно импульсов первой и второй ступеней на величину длительности импульса. Зависимость измеренного расщепления от интенсивности представлена на рис. 6. Экспериментальные данные хорошо согласуются с кривой (10) при значении коэффициента Эйнштейна второго перехода для компоненты 2-1 $A_{2-1}=8(1) \cdot 10^{5}$ s и отстройки частоты второго перехода $\Delta \lambda_{23}=45(5) \mathrm{MHz}$.

\section{Заключение}

Экспериментальные исследования показывают, что компоненты переходов трехступенчатой схемы фотоионизации существенно неоднородны по интенсивности, и сечения поглощения отличаются более чем на порядок. Это необходимо учитывать при оценке эффективности и селективности различных каналов фотоионизации. Отношения величин сечений компонент хорошо согласуются с классическими формулами, что позволило найти полные сечения всех переходов схемы фотоионизации для изотопов ${ }^{175} \mathrm{Lu},{ }^{176} \mathrm{Lu},{ }^{177} \mathrm{Lu}$ и ${ }^{177 m} \mathrm{Lu}$.

Эксперименты по исследованию расщепления переходов при сильно отличающихся частотах Раби позволили осуществить измерение частоты Раби и определить связь между измеренными эффективными сечениями поглощения и коэффициентом Эйнштейна. Таким образом, сечение поглощения второго перехода ${ }^{175} \mathrm{Lu} 1.6(3) \cdot 10^{-12} \mathrm{~cm}^{2}$ соответствует коэффициенту Эйнштейна $8(1) \cdot 10^{5} 1 / \mathrm{s}$.

\section{Финансирование работы}

Исследование выполнено за счет гранта Российского научного фонда (проект № 17-13-01180).

\section{Конфликт интересов}

Авторы заявляют, что у них нет конфликта интересов.

\section{Приложение}

Значения коэффициентов $C(F, J, \grave{F}, \grave{J}, I)$ из (1) рассчитываются по формулам для первого перехода $\vec{J}=J+1$ $(\grave{J}=5 / 2, J=3 / 2)$.

$$
\begin{aligned}
& \text { Для случая } \dot{F}=F-1 \\
& C(F, J, \grave{F}, \grave{J}, I)=(F+I-J-1)(F+I-J) \\
& \quad \times(I+J-F+1) \frac{(I+J-F+2)}{2 \cdot 2 F(2 F+1)(J+1)(2 J+3)} .
\end{aligned}
$$

Для случая $\grave{F}=F$

$$
\begin{gathered}
C(F, J, \grave{F}, \grave{J}, I)=(F-I+J)(F+I-J+1) \\
\quad \times(I+J+F+1) \frac{(I+J-F)}{2 F(2 F+2) J(2 J-1)} .
\end{gathered}
$$

Для случая $\grave{F}=F+1$

$$
\begin{aligned}
& C(F, J, \grave{F}, \grave{J}, I)=(F+I-J+1)(I+J-F) \\
& \quad \times(F+I-J+2) \frac{(I+J-F-1)}{2(2 F+1)(2 F+2) J(2 J-1)} .
\end{aligned}
$$

Для второго и третьего перехода $\vec{J}=J-1(\grave{J}=3 / 2$, $J=5 / 2),(\grave{J}=1 / 2, J=3 / 2)$. 
Для случая $\grave{F}=F-1$

$$
\begin{gathered}
C(F, J, \grave{F}, \grave{J}, I)=(F-I+J-1)(F-I+J) \\
\quad \times(F+I+J+1) \frac{(F+I+J)}{2 \cdot 2 F(2 F+1) J(2 J-1)} .
\end{gathered}
$$

Для случая $\grave{F}=F$

$$
\begin{gathered}
C(F, J, \grave{F}, \grave{J}, I)=(F-I+J)(F+I-J+1) \\
\times(I+J+F+1) \frac{(I+J-F)}{2 F(2 F+2) J(2 J-1)} .
\end{gathered}
$$

Для случая $\grave{F}=F+1$

$$
\begin{aligned}
& C(F, J, \grave{F}, \grave{J}, I)=(F+I-J+1)(I+J-F) \\
& \quad \times(F+I-J+2) \frac{(I+J-F-1)}{2(2 F+1)(2 F+2) J(2 J-1)} .
\end{aligned}
$$

Значения коэффициентов $C(F, J, \grave{F}, \grave{J}, I)$ для изотопов лютеция.

$$
{ }^{175} \mathrm{Lu},{ }^{177} \mathrm{Lu}, I=7 / 2 .
$$

\begin{tabular}{l|c|c}
\hline \multicolumn{2}{c}{ Первый переход $\grave{J}=J+1(\grave{J}=5 / 2, J=3 / 2)$} \\
\hline$F$ & $\grave{F}$ & $C(F, J, \grave{F}, \grave{J}, I)$ \\
\hline \multirow{3}{*}{2} & 1 & 0.4 \\
& 2 & 0.4 \\
3 & 3 & 0.2 \\
\hline \multirow{3}{*}{4} & 2 & 0.19 \\
& 3 & 0.417 \\
& 4 & 0.393 \\
\hline \multirow{3}{*}{5} & 3 & 0.083 \\
& 4 & 0.33 \\
& 5 & 0.587 \\
\hline & 4 & 0.025 \\
& 6 & 0.187 \\
\end{tabular}

\begin{tabular}{c|c|c}
\hline \multicolumn{3}{c}{ Второй переход $\grave{J}=J-1(\grave{J}=3 / 2, J=5 / 2)$} \\
\hline$F$ & $\dot{F}$ & $C(F, J, \dot{F}, \dot{J}, I)$ \\
\hline 1 & 2 & 1 \\
\hline 2 & 2 & 0.6 \\
& 3 & 0.4 \\
\hline 3 & 2 & 0.214 \\
& 3 & 0.625 \\
& 4 & 0.161 \\
\hline 4 & 3 & 0.458 \\
& 4 & 0.495 \\
& 5 & 0.047 \\
\hline 5 & 4 & 0.72 \\
& 5 & 0.28 \\
\hline 6 & 5 & 1 \\
\hline
\end{tabular}

\begin{tabular}{c|c|c}
\hline \multicolumn{3}{c}{ Третий переход $J=J-1(J=1 / 2, J=3 / 2)$} \\
\hline$F$ & $F$ & $C(F, J, F, J, I)$ \\
\hline 2 & 3 & 1 \\
\hline 3 & 3 & 0.75 \\
& 4 & 0.25 \\
\hline 4 & 3 & 0.417 \\
& 4 & 0.583 \\
\hline 5 & 4 & 1 \\
\hline
\end{tabular}

$$
{ }^{176} \mathrm{Lu}, I=7 .
$$

\begin{tabular}{c|c|c}
\hline \multicolumn{3}{c}{ Первый переход $\grave{J}=J+1(\grave{J}=5 / 2, J=3 / 2)$} \\
\hline$F$ & $\dot{F}$ & $C(F, J, \grave{F}, \grave{J}, I)$ \\
\hline 5.5 & 4.5 & 0.556 \\
& 5.5 & 0.328 \\
& 6.5 & 0.116 \\
\hline 6.5 & 5.5 & 0.29 \\
& 6.5 & 0.418 \\
& 7.5 & 0.291 \\
\hline 7.5 & 6.5 & 0.13 \\
& 7.5 & 0.367 \\
& 8.5 & 0.503 \\
\hline 8.5 & 7.5 & 0.04 \\
& 8.5 & 0.22 \\
& 9.5 & 0.741 \\
\hline
\end{tabular}

\begin{tabular}{c|c|c}
\hline \multicolumn{3}{c}{ Второй переход $\grave{J}=J-1(\grave{J}=3 / 2, J=5 / 2), I=7$} \\
\hline$F$ & $\grave{F}$ & $C(F, J, \grave{F}, \grave{J}, I)$ \\
\hline 4.5 & 5.5 & 1 \\
\hline 5.5 & 5.5 & 0.492 \\
& 6.5 & 0.507 \\
\hline 6.5 & 5.5 & 0.149 \\
& 6.5 & 0.627 \\
& 7.5 & 0.551 \\
\hline 7.5 & 6.5 & 0.383 \\
& 7.5 & 0.551 \\
& 8.5 & 0.067 \\
\hline 8.5 & 7.5 & 0.671 \\
& 8.5 & 0.329 \\
\hline 9.5 & 8.5 & 1 \\
\hline
\end{tabular}

\begin{tabular}{c|c|c}
\hline \multicolumn{3}{c}{ Третий переход $\grave{J}=J-1(\grave{J}=1 / 2, J=3 / 2), I=7$} \\
\hline$F$ & $\dot{F}$ & $C(F, J, \dot{F}, \dot{J}, I)$ \\
\hline 5.5 & 6.5 & 1 \\
\hline 6.5 & 6.5 & 0.711 \\
& 7.5 & 0.289 \\
\hline 7.5 & 6.5 & 0.378 \\
& 7.5 & 0.622 \\
\hline 8.5 & 7.5 & 1 \\
\hline
\end{tabular}


${ }^{177 m} \mathrm{Lu}, I=23 / 2$

\begin{tabular}{c|c|c}
\hline \multicolumn{3}{c}{ Первый переход $\grave{J}=J+1(\grave{J}=5 / 2, J=3 / 2)$} \\
\hline$F$ & $\dot{F}$ & $C(F, J, \dot{F}, \grave{J}, I)$ \\
\hline 10 & 9 & 0.603 \\
& 10 & 0.303 \\
& 11 & 0.094 \\
\hline 11 & 10 & 0.332 \\
& 11 & 0.414 \\
& 12 & 0.254 \\
\hline 12 & 11 & 0.154 \\
& 12 & 3.367 \\
& 13 & 0.465 \\
\hline 13 & 12 & 0.048 \\
& 13 & 0.236 \\
& 14 & 0.716
\end{tabular}

\begin{tabular}{c|c|c}
\hline \multicolumn{3}{c}{ Второй переход $\grave{J}=J-1(\grave{J}=3 / 2, J=5 / 2)$} \\
\hline$F$ & $\grave{F}$ & $C(F, J, \grave{F}, \grave{J}, I)$ \\
\hline 9 & 10 & 1 \\
\hline 10 & 10 & 0.455 \\
& 11 & 0.545 \\
\hline 11 & 10 & 0.128 \\
& 11 & 0.62 \\
& 12 & 0.251 \\
\hline 12 & 11 & 0.351 \\
& 12 & 0.571 \\
& 13 & 0.078 \\
\hline 13 & 12 & 0.646 \\
& 13 & 0.354 \\
\hline 14 & 13 & 1 \\
\hline
\end{tabular}

\begin{tabular}{c|c|c}
\hline \multicolumn{3}{c}{ Третий переход $\grave{J}=J-1(\grave{J}=1 / 2, J=3 / 2)$} \\
\hline$F$ & $\vec{F}$ & $C(F, J, \dot{F}, \vec{J}, I)$ \\
\hline 10 & 11 & 1 \\
\hline 11 & 11 & 0.694 \\
& 12 & 0.306 \\
\hline 12 & 11 & 0.361 \\
& 12 & 0.639 \\
\hline 13 & 12 & 1 \\
\hline
\end{tabular}

\section{Список литературы}

[1] Bhardwaj R., VanDerMeer A., Das S.K., DeBruin M., Gascon J., Wolterbeek H.T., Denkova A.G., Serra-Crespo P. // Sci. Rep. 2017. V. 7. P. 44242. doi 10.1038/srep44242

[2] D'yachkov A.B., Firsov V.A., Gorkunov A.A., Labozin A.V., Mironov S.M., Panchenko V.Y., Semenov A.N., Shatalova G.G., Tsvetkov G.O. // Appl. Phys. B. 2015. V. 121. N 4. P. 425. doi 10.1007/s00340-015-6248-0

[3] D'yachkov A.B., Gorkunov A.A., Labozin A.V., Mironov S.M., Tsvetkov G.O., Panchenko V.Y., Firsov V.A. // Opt. Spectrosc. 2018. V. 125. N 6. P. 839. doi 10.1134/S0030400X19020127
[4] Ageeva I.V., D'yachkov A.B., Gorkunov A.A., Labozin A.V., Mironov S.M., Panchenko V.Y., Firsov V.A., Tsvetkov G.O., Tsvetkova E.G. // Quant. Electron. 2019. V. 49. N 9. P. 832. doi 10.1070/QEL17049

[5] Axner O., Gustavson J., Omenetto N., Winefordner J. // Spectrochim. Acta. B. 2004. V. 59. N 1. P. 1. doi 10.1016/j.sab.2003.10.002

[6] D'yachkov A.B., Gorkunov A.A., Labozin A.V., Mironov S.M., Panchenko V.Y., Firsov V.A., Tsvetkov G.O. // Instrum. Exp. Tech. 2018. V. 61. N 4. P. 548. doi 10.1134/S0020441218040048

[7] Dyachkov A.B., Gorkunov A.A., Labozin A.V., Mironov S.M., Panchenko V.Y., Firsov V.A., Tsvetkov G.O. // Quant. Electron. 2018. V. 48. N 11. P. 1043. doi 10.1070/QEL16793

[8] Petersen F.R., Shugart H.A. // Phys. Rev. 1962. V. 126. N 1. P. 252. doi 10.1103/PhysRev.126.252

[9] Brenner T., Büttgenbach S., Rupprecht W., Träber F. // Nucl. Phys. A. 1985. V. 440. N 3. P. 407. doi 10.1016/0375-9474(85)90237-4

[10] Shore B.W., Ackerhalt J.R. // Phys. Rev. A. 1977. V. 15. N 4. P. 1640. doi 10.1103/PhysRevA.15.1640

[11] Ackerhalt J.R., Eberly J.H., Shore B.W. // Phys. Rev. A. 1979. V. 19. N 1. P. 248. doi 10.1103/PhysRevA.19.248

[12] Ackerhalt J.R., Eberly J.H. // Phys. Rev. A. 1976. V. 14. N 5. P. 1705. doi 10.1103/PhysRevA.14.1705 